\title{
RACSAM
}

Rev. R. Acad. Cien. Serie A. Mat.

VOL. 103 (2), 2009, pp. 353-372

Matemática Aplicada / Applied Mathematics

Artículo panorámico / Survey

\section{Applications of fluid flow matrix analytic methods in ruin theory -a review}

\author{
Andrei L. Badescu and David Landriault
}

\begin{abstract}
In this paper, we present a unified probabilistic approach to analyze a wide class of insurance risk models in a ruin theoretical context. Contrary to the traditional analytic approach mainly encountered in the literature, this alternative approach is based on matrix analytic methods (MAMs) that have become an increasingly popular set of tools in the study of various applied probability models. We make use of the recent advances in the study of fluid queues to analyze some insurance risk processes and their ruin related quantities. The advantages and disadvantages of MAMs over alternative methods are also discussed.
\end{abstract}

\section{Métodos analíticos matriciales para flujos fluidos aplicados a la teoría de la ruina —una revisión}

\begin{abstract}
Resumen. Este artículo presenta un enfoque probabilístico unificador para el análisis de una clase amplia de modelos de riesgo en el contexto de la teoría de la ruina. Contrastando con el enfoque analítico tradicional que domina en la literatura, nuestro enfoque alternativo está basado en métodos analíticos matriciales (MAMs) que progresivamente han ido haciéndose populares herramientas en el estudio de diversos modelos de probabilidad aplicada. Utilizamos los últimos avances en el estudio de colas fluidas para analizar algunos procesos de riesgo de seguros y sus variables de ruina asociadas. Discutimos de las ventajas y desventajas de los MAMs en comparación a los métodos alternativos.
\end{abstract}

\section{Introduction}

Matrix analytic methods (MAMs) are a set of powerful tools developed to analyze a wide variety of stochastic models that arise in telecommunications, operations research, management science, industrial engineering, computer engineering, bio-statistics, to name a few. The main focus of this review paper is to present a recent area of applications of MAMs, namely ruin theory. By making use of the connection between an insurer's surplus process and a particular fluid queue, we present a unified methodology for studying a large class of insurance risk models via the recent developments in MAMs for the analysis of fluid queues.

As their name suggests, MAMs are based on matrix calculations that often have nice probabilistic interpretations. The elegant structural forms of these quantities provide algorithmic tractability, a feature that most of the traditional analytic techniques do not possess. Furthermore, MAMs make no use of the theory of eigenvalues. Indeed, this is in total accordance with the fact that the fundamental matrices have

Presentado por / Submitted by José Garrido.

Recibido / Received: 2 de febrero de 2009. Aceptado / Accepted: 6 de mayo de 2009.

Palabras clave / Keywords: Matrix analytic methods, fluid queue, first passage probabilities, Markovian arrival process, phase-type distribution, surplus process.

Mathematics Subject Classifications: 60J75, 60J25, 60G55.

(C) 2009 Real Academia de Ciencias, España. 
probabilistic meanings, as the eigenvalues do not. As will be shown, the applications of MAMs in ruin theory allow the derivation of an expression for certain ruin-related quantities without directly involving the roots of the so-called Lundberg fundamental equation. The reader is referred to e.g. Badescu and Breuer [19, (2008)] for a brief discussion of numerical instability sometimes encountered in the calculation of these roots. The role of the Lundberg equation roots in the traditional analytic approach is replaced in MAMs by a matrix quantity representing the Laplace-Stieljes transform (LST) of a busy period in a particular fluid queue. Due to its importance, the LST of this busy period will be the subject matter of Section 2.3.1.

But first, two simple examples of MAMs, namely the phase-type distribution (see e.g. Neuts [51, (1978)]) and the Markovian arrival process (see e.g. Neuts [52, (1979)]), are briefly discussed in the following two sub-sections.

\subsection{Phase-type distribution}

The phase-type distribution was introduced by Neuts [51, (1978)] as a generalization of the exponential distribution. Let $\mathcal{Z}=\left\{Z_{t}: t \geq 0\right\}$ be a time-homogeneous continuous-time Markov chain (CTMC) with state space $\{1, \ldots, n, n+1\}$ and infinitesimal generator

$$
\mathbf{Q}=\left[\begin{array}{ll}
\mathbf{B} & \mathbf{b} \\
\mathbf{0} & 0
\end{array}\right]
$$

where $\mathbf{B}$ is a $n \times n$ square matrix and $\mathbf{b}=-\mathbf{B e}$. Throughout this paper, $\mathbf{e}$ is a column vector of $1 \mathrm{~s}$ while $\mathbf{0}$ is a matrix of $0 \mathrm{~s}$, both of appropriate dimensions. The initial probability vector of the CTMC $\mathcal{Z}$ is denoted by $\left(\beta, \beta_{n+1}\right)$ where $\beta$ is a row vector of size $n$. The states $\{1, \ldots, n, n+1\}$ of the CTMC $\mathcal{Z}$ are referred to as phases. Let $T=\inf \left\{t \geq 0, Z_{t}=n+1\right\}$ be the time until absorption of the CTMC $\mathcal{Z}$ in state $n+1$. The distribution of $T$ is called phase-type $(\mathbf{P H})$ distribution with parameters $(\beta, \mathbf{B})$.

From its construction, it is easy to see that the PH random variable (r.v.) $T$ consists of a collection of exponentially distributed time segments among the phase changes of the CTMC $\mathcal{Z}$. Phase-type distributions possess several properties that make them a versatile class of r.v.'s, some of them being listed below without proof. The interested reader is referred to Neuts [53, (1981)] for a detailed description.

Theorem 1 For a PH r.v. T with representation $(\beta, \mathbf{B})$,

(a) the cumulative distribution function (c.d.f.) is given by $F(t)=1-\beta \mathrm{e}^{\mathbf{B} t} \mathbf{e}$;

(b) the probability density function (p.d.f.) is given by $f(t)=\beta \mathrm{e}^{\mathbf{B} t} \mathbf{b}$;

(c) the moment generating function (m.g.f.) is given by $\tilde{F}(s)=\int_{0}^{\infty} \mathrm{e}^{s t} \mathrm{~d} F(t)=\beta(-s \mathbf{I}-\mathbf{B})^{-1} \mathbf{b}+\beta_{n+1}$.

It is easy to observe that the phase-type distribution is a matrix generalization of the exponential distribution. Indeed, by letting $\mathbf{B}=-\lambda$ and $\beta=1$, one recovers an exponential r.v. with mean $1 / \lambda$. Mixtures of exponentials/Erlangs are other special cases of this large class of distribution functions.

Proposition 1 The class of PH distributions is closed under the formation of finite mixtures, finite convolutions, finite minima and maxima, and compounding with discrete phase-type distributions.

Proposition 2 The class of PH distributions is dense in the sense of weak convergence in the class of all distributions with positive support.

\subsection{Markovian arrival process}

A second example of MAMs is a versatile class of point processes known as the Markovian arrival process (MAP). A $m$-dimensional MAP is defined through two processes: a continuous-time Markov process $\mathcal{J}=\{J(t): t \geq 0\}$ with state space $S_{1}=\{1, \ldots, m\}$ and a counting process $\mathcal{N}=\{N(t), t \geq 0\}$ with 
state space $\{0,1, \ldots\}$. For the counting process $\mathcal{N}$, the number of marked transitions of the CTMC $\mathcal{J}$ by time $t$ is represented by the r.v. $N(t)$. The transition rates which are not marked are described by the matrix $\mathbf{D}_{0}$, while those that are marked are described by the matrix $\mathbf{D}_{1}$. The initial state distribution is given by the row vector $\delta^{+}$. The matrices $\mathbf{D}_{0}$ and $\mathbf{D}_{1}$ satisfy the following conditions:

i. $\mathbf{D}_{0}+\mathbf{D}_{1}$ is the infinitesimal generator of an irreducible CTMC $\mathcal{J}$ (the so-called environmental process) on the state space $S_{1}$;

ii. $\mathbf{D}_{0}[i, i]<0$ for all $i$, and $\mathbf{D}_{0}[i, j] \geq 0$ for all $i \neq j$ with $i, j \in S_{1}$;

iii. $\mathbf{D}_{1}[i, j] \geq 0$ for all $i, j \in S_{1}$;

iv. for $i \neq j, \mathbf{D}_{0}[i, j]$ is the rate at which a change of the underlying phase from $i$ to $j$ occurs without an arrival (unmarked transition);

v. $\mathbf{D}_{1}[i, j]$ is the rate at which a change of the underlying phase from $i$ to $j$ occurs with an arrival (marked transition) (here, $j=i$ is allowed).

For a fuller description the interested reader is referred to Latouche and Ramaswami [44, (1999)].

As has been shown by Asmussen and Koole [14, (1993)], it is possible to model point processes with a fairly general degree of complexity by MAPs. For this reason (and many others), MAPs have become extensively used in queueing theory and performance evaluation. Several procedures have been developed over time to estimate the transition matrices $\mathbf{D}_{0}$ and $\mathbf{D}_{1}$ from observed data. The so-called EM algorithm has proven to be a good mean of approximating the maximum likelihood estimator (see e.g. Asmussen et al. $[15,(1996)])$.

Also, several well-known point processes are special cases of the general MAP:

(a) The Poisson process with interarrival rate $\lambda$ is a particular MAP with $\delta^{+}=1, \mathbf{D}_{0}=-\lambda$ and $\mathbf{D}_{1}=\lambda$.

(b) The renewal point process with $\operatorname{PH}(\beta, \mathbf{B})$ sojourns between arrivals is a MAP with $\delta^{+}=\beta, \mathbf{D}_{0}=\mathbf{B}$ and $\mathbf{D}_{1}=\mathbf{b} \beta$.

(c) The Markov-modulated Poisson process (MMPP) - consider an underlying Markov process with a finite number of states, say $m$. Assume that while the system is in state $i$, claims arrive according to a Poisson process at rate $\lambda_{i}(i=1, \ldots, m)$. Denoting the underlying Markov process generator by $\boldsymbol{\Gamma}$, the MAP representation is given by $\mathbf{D}_{1}=\operatorname{diag}\left\{\lambda_{1}, \ldots, \lambda_{m}\right\}$ and $\mathbf{D}_{0}=\mathbf{\Gamma}-\mathbf{D}_{1}$.

(d) The PH semi-Markov process - consider $m$ independent $\mathrm{PH}$ distributions: $F_{j} \sim \mathrm{PH}\left(\beta_{j}, \mathbf{B}_{j}\right)$ with exit rate vectors $\mathbf{b}_{j}=-\mathbf{B}_{j} \mathbf{e}$ for $j=1, \ldots, m$. Consider also a background discrete-time Markov chain $\{X(n)\}_{n \geq 0}$ with $m$ states and let $p_{i j}$ be its one-period transition probability from state $i$ to state $j$. Consider a semi-Markov process for which the $n$-th interarrival time has distribution $F_{j}$ when $X(n)=j$. The MAP representation of the formulated PH semi-Markov process is

$$
\mathbf{D}_{0}=\left[\begin{array}{ccccc}
\mathbf{B}_{1} & \mathbf{0} & \mathbf{0} & \ldots & \mathbf{0} \\
\mathbf{0} & \mathbf{B}_{2} & \mathbf{0} & \ldots & \mathbf{0} \\
\vdots & \vdots & \vdots & \ddots & \vdots \\
\mathbf{0} & \mathbf{0} & \mathbf{0} & \ldots & \mathbf{B}_{m}
\end{array}\right], \quad \mathbf{D}_{1}=\left[\begin{array}{cccc}
p_{11} \mathbf{b}_{1} \beta_{1} & p_{12} \mathbf{b}_{1} \beta_{2} & \ldots & p_{1 m} \mathbf{b}_{1} \beta_{m} \\
\vdots & \vdots & \ddots & \vdots \\
p_{m 1} \mathbf{b}_{m} \beta_{1} & p_{m 2} \mathbf{b}_{m} \beta_{2} & \ldots & p_{m m} \mathbf{b}_{m} \beta_{m}
\end{array}\right]
$$

In the next sub-section, we define the class of risk models subject to analysis via MAMs in this paper. 


\subsection{The insurance risk model}

In this paper, we consider an insurer for which its surplus process $\underline{R}=\{R(t), t \geq 0\}$ is defined as

$$
R(t)=u+c t-\sum_{k=1}^{N(t)} X_{k},
$$

where $u$ is the insurer's initial surplus and $c$ is the incoming premium rate. The claim number process $\{N(t), t \geq 0\}$ is assumed to have interclaim times $T_{k}$ for $k=1,2, \ldots$ Also, $X_{k}$ denotes the size of the $k$-th claim $(k=1,2, \ldots)$.

Traditionally, it has been assumed that the interclaim times $\left\{T_{k}\right\}_{k=1}^{\infty}$ and the claim sizes $\left\{X_{k}\right\}_{k=1}^{\infty}$ are mutually independent r.v.'s. Despite the fact that this independence assumption leads to less realistic insurance risk models, risk models of this kind which include the compound Poisson risk model and the Sparre Andersen risk model are analytically tractable (for a detailed description, see Asmussen [11, (2000)]). With the advance in analytic tools, a new trend has emerged to identify and study risk models which relax the stringent independence assumptions of the Sparre Andersen risk model. Among others, a popular class of generalizations is the class of risk models for which the increments of the surplus process from the time of a claim to its subsequent are all independent (as well as identically distributed), but where the r.v.'s $T_{k}$ and $X_{k}$ are possibly dependent for any $k=1,2, \ldots$. Risk models that belong to this class preserve the random structure of the traditional Sparre Andersen risk model. The interested reader is referred to e.g. Albrecher and Teugels [9, (2006)], Boudreault et al. [28, (2006)] and Cossette et al. [34, (2008)] for some examples.

In this paper, we consider a different class of generalizations. We assume that the claim number process $\{N(t), t \geq 0\}$ is a MAP with representation $\left(\delta^{+}, \mathbf{D}_{0}, \mathbf{D}_{1}\right)$. We also assume that the claim amounts are PH distributed. More precisely, for a transition of the CTMC $\mathcal{J}$ from state $i$ to state $j$ at the time of a claim (i.e. marked transition), we suppose that the accompanying claim size has a PH distribution with parameters $\left(\beta_{i j}, \mathbf{B}_{i j}\right)$. Without loss of generality, we assume that all the PH claim size distributions are of size $n$. Even if the class of phase-type distributions is dense among the distributions defined on the non-negative real line, one should keep in mind that PH distributions are by definition light-tailed. Hence a fit to a heavytailed distribution might be poor (especially in the right-hand tail) even for a phase-type distribution with a relatively large number of phases.

Under these assumptions, we have constructed a very general non-renewal insurance risk model where claim sizes and interclaim times are all dependent r.v.'s. In addition, many risk models are special cases of the MAP risk model described above, namely the Sparre Andersen risk model with PH claim sizes and PH interclaim times, as well as certain dependent risk models such as the MMPP with PH claim sizes (e.g. Lu and Li [49, (2009)]) and Albrecher and Boxma's [6, (2005)] semi-Markov risk model with PH claim sizes. These risk models (without the PH assumption for the claim sizes) have generally been analyzed using the traditional analytic method. In this paper, we review a recently new methodology as a viable alternative to analyze these risk models. The use of MAMs together with the existing connections between risk processes and fluid flow processes allow the analysis of these risk processes in a unified and relatively straightforward way. The next section of the paper will familiarize the reader with some fluid flow models of interest and their most relevant properties for the study of the surplus process $\underline{R}$.

\section{Fluid flow models}

Motivated by their applications in several applied probability models, the theory of fluid flows has been an active area of research in recent years. One of the most common applications of these stochastic processes is in telecommunication where fluid flows are used to model the traffic evolution in a communication channel. Another simple example of a fluid flow is given by an infinite capacity buffer with inflow and outflow rates controlled by a Markov chain. When the buffer is empty, it remains empty until the controlling Markov chain moves to a state with a net inflow rate. Once this happens, the buffer level increases until the time at which the underlying Markov chain transits to a phase with a net outflow rate. 
In this section, we define a Markov modulated fluid flow (MMFF) and later establish its connection with the MAP risk process described in Section 1.3. Towards the end of the section, we review some fundamental fluid flow first passage probabilities that are central to the analysis of ruin-related quantities in Section 3.

\subsection{The Markov modulated fluid flow model}

Let $(\mathcal{F}, \mathcal{W})=\{(F(t), W(t)): t \geq 0\}$ be a MMFF where $F(t)$ is the level of a fluid buffer at time $t$ and the environmental process $\mathcal{W}$ is a CTMC with state space $S=S_{1} \cup S_{2} \cup S_{3}$ and infinitesimal generator T partitioned as

$$
\mathbf{T}=\left(\begin{array}{ccc}
\mathbf{T}_{11} & \mathbf{T}_{12} & \mathbf{T}_{13} \\
\mathbf{T}_{21} & \mathbf{T}_{22} & \mathbf{T}_{23} \\
\mathbf{T}_{31} & \mathbf{T}_{32} & \mathbf{T}_{33}
\end{array}\right)
$$

The submatrices $\mathbf{T}_{i j}(i, j=1,2,3)$ contain the rates of transition from phases in $S_{i}$ to phases in $S_{j}$. The fluid level process $\mathcal{F}=\{F(t): t \geq 0\}$ is described as follows: during sojourn of $\mathcal{W}$ in state $i \in S_{1}$, the fluid level increases at rate $c_{i}>0$; during sojourn of $\mathcal{W}$ in state $i \in S_{2}$, the fluid level decreases at rate $c_{i}>0$; during sojourn of $\mathcal{W}$ in state $i \in S_{3}$, the fluid level remains constant (i.e. $c_{i}=0$ ). Mathematically, the evolution of the fluid flow can be described through the piecewise linear function

$$
L(t)=u+\int_{0}^{t} c_{W(v)} \mathrm{d} v,
$$

where $u$ is the fluid level at time 0 . The fluid level is then given by

$$
F(t)=L(t)-\min (0, \tilde{L}(t))
$$

where $\tilde{L}(t)=\min _{0 \leq v \leq t} L(v)$. We denote the canonical fluid flow model $\mathcal{F}$ as $\mathcal{F}=\mathcal{F}\left(\mathbf{T}, \mathbf{C}_{1},-\mathbf{C}_{2}\right)$ where $\mathbf{C}_{1}=\operatorname{diag}\left(c_{i}, i \in S_{1}\right)$ and $\mathbf{C}_{2}=\operatorname{diag}\left(c_{i}, i \in S_{2}\right)$ are two diagonal matrices containing the rates of increase and decrease of the fluid flow $\mathcal{F}$ respectively.

Three variants of the fluid flow $\mathcal{F}$ are particularly useful in the analysis of ruin-related quantities for the surplus process $\underline{R}$ :

Finite Buffer Fluid Flow: In relation with the fluid flow $\mathcal{F}$, we define the finite buffer fluid flow ${ }^{b} \mathcal{F}=$ ${ }^{b} \mathcal{F}\left(\mathbf{T}, \mathbf{C}_{1},-\mathbf{C}_{2}\right)$ which allows the fluid level to decrease only when it is positive and to increase only when it is less than a buffer level $b>0$.

Reflected Fluid Flow: Associated to the fluid flow $\mathcal{F}$, we also define the reflected fluid flow $\mathcal{F}^{r}$ obtained by reversing the roles of the up and down environment states. Mathematically, we write $\mathcal{F}^{r}=$ $\mathcal{F}\left(\mathbf{T}, \mathbf{C}_{2},-\mathbf{C}_{1}\right)$.

Reflected Finite Buffer Fluid Flow: In a similar manner, we define the reflected finite buffer fluid flow ${ }^{b} \mathcal{F}^{r}={ }^{b} \mathcal{F}\left(\mathbf{T}, \mathbf{C}_{2},-\mathbf{C}_{1}\right)$ which is the reflection of the fluid queue ${ }^{b} \mathcal{F}$.

\subsection{Connection to risk processes}

Due to their relatively proximity, several authors —Asmussen [10, (1995)] being the first among themanalyze risk processes via fluid flows. For instance, the $\operatorname{MMFF}(\mathcal{F}, \mathcal{W})$ can be used to analyze a large class of risk processes (see e.g. Ahn et al. [2, (2007)], Badescu et al. [20, (2005)] and Ramaswami [55, (2006)] for several illustrative examples). The main purpose of this subsection is to show how the MMFF $(\mathcal{F}, \mathcal{W})$ can be used to analyze the surplus process $\underline{R}$ described in Section 1.3. Some adjustments to the general $\operatorname{MMFF}(\mathcal{F}, \mathcal{W})$ are first required to match the sample paths of the two processes.

The first modification relates to the accumulation/depletion rates $c_{i}$ of the $\operatorname{MMFF}(\mathcal{F}, \mathcal{W})$. Given that premiums are collected at a constant rate $c$ in the surplus process $\underline{R}$, we shall assume that the linear rates of 
increase/decrease of the fluid flow $\mathcal{F}$ do not dependent on the underlying phase of the CTMC $\mathcal{W}$ (i.e. $c_{i}=c$ for all $i$ ). As a consequence, the diagonal matrices $\mathbf{C}_{1}$ and $\mathbf{C}_{2}$ will be replaced by the scalar $c$. The second modification pertains to the fact that the surplus process $\underline{R}$ does not stay at a constant level over time. Therefore, we shall assume that the set of phases $S_{3}$ of the $\operatorname{MMFF}(\mathcal{F}, \mathcal{W})$ is empty. Based on those two adjustments, the resulting MMFF is denoted by $\left(\underline{F_{c}}, \underline{W}\right)=\left\{F_{c}(t), W(t)\right\}$ where the CTMC $\underline{W}$ has state space $S=S_{1} \cup S_{2}$ and infinitesimal generator

$$
\mathbf{T}=\left(\begin{array}{ll}
\mathbf{T}_{11} & \mathbf{T}_{12} \\
\mathbf{T}_{21} & \mathbf{T}_{22}
\end{array}\right)
$$

Note that the accumulation/depletion rates of the fluid flow $F_{c}$ is its index $c$.

Finally, paths of the surplus process $\underline{R}$ before ruin can be obtained from segments of the fluid process $\left(F_{c}, \underline{J}\right)$ before the fluid level becomes empty by replacing the downward linear paths of the fluid flow by downward jumps of appropriate sizes. To do so, one has to interpret a claim of size $x$ as being paid continuously at rate $c$ over a (unobserved) time interval of length $x / c$. This artifice changes the clock time in the fluid model by extracting all the spurious time intervals of downward linear descents (replacing them with instantaneous downward jumps). A sample path of these two processes is illustrated in Figure 1.

To analyze the surplus process $\underline{R}$, it remains to specify the parametrization of the fluid flow $\left(\underline{F_{c}}, \underline{W}\right)$ with respect to its generator $\mathbf{T}$ and its state space $S$. We recall that, for the surplus process $\underline{R}$, claims arrive according to a MAP $\left(\delta^{+}, \mathbf{D}_{0}, \mathbf{D}_{1}\right)$. A claim that occurs at the time of a transition of the underlying CTMC from state $i$ to state $j$ is $\operatorname{PH}\left(\beta_{i j}, \mathbf{B}_{i j}\right)$ distributed. Let $m$ be the dimension of the set $S_{1}$ and $n$ be the dimension of all the $\mathrm{PH}$ claim size distributions. Hence, the associated fluid flow model $\left(F_{c}, \underline{J}\right)$ has state space $S=S_{1} \cup S_{2}$ where the dimensions of $S_{1}$ and $S_{2}$ are $m$ and $m \times n \times m$ respectively. The $(i, j)$-th element of the infinitesimal generator (1) are:

$$
\begin{aligned}
\mathbf{T}_{11}[i, j] & =\mathbf{D}_{0}[i, j], \\
\mathbf{T}_{12}[i,(i, j, k)] & =\mathbf{D}_{1}[i, j] \beta_{i j}[k], \\
\mathbf{T}_{21, c}[(i, j, r), j] & =-c \sum_{s=1}^{n} \mathbf{B}_{i j}[r, s], \\
\mathbf{T}_{22, c}[(i, j, r),(i, j, s)] & =c \mathbf{B}_{i j}[r, s] .
\end{aligned}
$$

Note that the matrices $\mathbf{T}_{21, c}$ and $\mathbf{T}_{22, c}$ contain the premium rate $c$ due to the scaling of the time interval over which claim occurs continuously at rate $c$ (as discussed above) — see Ramaswami [55, (2006)] for more details. We also define the absorbing rate vector $\mathbf{t}_{21, c}$ as $\mathbf{t}_{21, c}=\mathbf{T}_{21, c} \mathbf{e}$.

We conclude the present section with some important relationships between first passage times in the fluid flow $F_{c}$ and the risk process $\underline{R}$ which can be found in e.g. Ramaswami [55, (2006)]. To this end, let ${ }_{a}^{z} \sigma_{c}(x, y)$ be the first passage time of the fluid level $\underline{F_{c}}$ from level $x$ to level $y$ avoiding a visit to the levels in $[0, a] \cup[z, \infty)$ enroute. Note that the arguments $\bar{a}$ and/or $z$ may be suppressed in the above definition whenever they are meaningless. For instance, we use ${ }_{0} \sigma_{c}(0, x)$ instead of ${ }_{0}^{x} \sigma_{c}(0, x)$ given that the fluid flow process has to reach level $x$ before any level in $[x, \infty)$. Similarly, let ${ }_{a}^{z} \tau_{c}(x, y)$ be the equivalent time in the risk process $\underline{R}$ (which is obtained from ${ }_{a}^{z} \sigma_{c}(x, y)$ by eliminating the time intervals in which the fluid flow $\underline{F_{c}}$ decreases over this first passage time).

Remark 1 A comparison of some first passage times in the fluid flow $\underline{F_{c}}$ and the risk process $\underline{R}$ yields the following observations:

(a) the first passage time from level 0 to level 0 in the risk model $\underline{R}$ is just half of the equivalent time in the fluid flow $\underline{F_{c}}$, i.e.

$$
\tau_{c}(0,0)=\frac{\sigma_{c}(0,0)}{2} .
$$



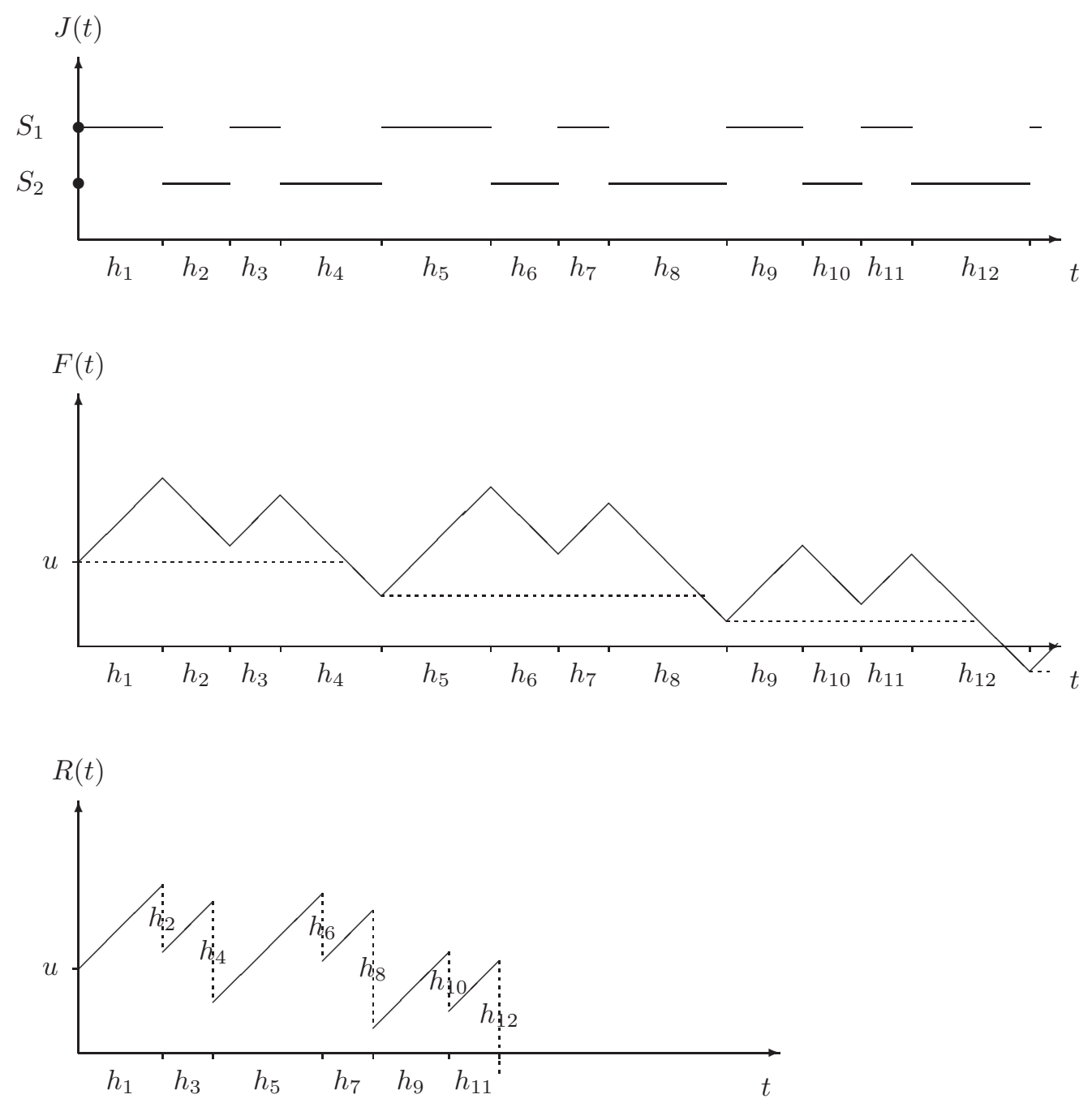

Figure 1. Connection between risk processes and fluid processes

(b) for the interval of descent from $\left(x, S_{2}\right)$ to $\left(y, S_{2}\right)$ with $0 \leq y<x$, we have

$$
\tau_{c}(x, y)=\frac{\sigma_{c}(x, y)}{2}-\frac{x-y}{2 c} .
$$

(c) for the interval of ascent from $\left(x, S_{1}\right)$ to $\left(y, S_{1}\right)$ with $0 \leq x<y$, we have

$$
\tau_{c}(x, y)=\frac{\sigma_{c}(x, y)}{2}+\frac{y-x}{2 c} .
$$

\subsection{First passage probabilities}

\subsubsection{The Laplace-Stieljes transform of the busy period}

Fundamental to the subsequent analysis is the LST of the busy period $\sigma_{c}(0,0)$ in the fluid queue $\underline{F_{c}}$ (see e.g. Ahn and Ramaswami [3, (2004)], Bean et al. [27, (2005)]). Let $\Psi_{c}(\delta)$ be this LST, a matrix arising due 
to the relevance of the initial and terminal states of the CTMC $\underline{W}$. More precisely, $\Psi_{c}(\delta)$ is a $\left|S_{1}\right| \times\left|S_{2}\right|$ matrix with $(i, j)$-element

$$
\left[\boldsymbol{\Psi}_{c}(\delta)\right]_{i j}=E\left[\mathrm{e}^{-\delta \sigma_{c}(0,0)} I\left(W\left(\sigma_{c}(0,0)\right)=j\right) \mid W(0)=i\right], \quad i \in S_{1}, \quad j \in S_{2},
$$

where $I(A)$ is the indicator function of the event $A$. Note that, for $\delta=0$, the scalar $\delta^{+} \boldsymbol{\Psi}_{c}(0)$ e corresponds to the probability that the fluid queue $\underline{F_{c}}$ will ever become empty given that $F_{c}(0)=0$. Clearly, this also corresponds to the infinite-time ruin probability in the associated risk model $\underline{R}$ when the initial surplus is 0 . Given that $\Psi_{c}(\delta)$ plays a central role in the transient analysis of the fluid flow $F_{c}$ (and implicitly in the associated surplus process $\underline{R}$ ), additional results pertaining to $\Psi_{c}(\delta)$ are presented below.

Rogers [58, (1994)] showed that $\Psi_{c}(\delta)$ (with $\delta \geq 0$ ) can be obtained from the minimal nonnegative solution of the Riccati equation

$$
\mathbf{T}_{12}+\left(\mathbf{T}_{11}-\delta \mathbf{I}\right) \mathbf{X}+\mathbf{X}\left(\mathbf{T}_{22, c}-\delta \mathbf{I}\right)+\mathbf{X} \mathbf{T}_{21, c} \mathbf{X}=\mathbf{0}
$$

with I being an identity matrix of appropriate dimensions. Later, Ramaswami [54, (1999)] proved that the minimal nonnegative solution of the matrix quadratic equation

$$
\mathbf{Y}=\mathbf{A}_{2}+\mathbf{A}_{1} \mathbf{Y}+\mathbf{A}_{0} \mathbf{Y}^{2}
$$

with

$$
\begin{aligned}
& \mathbf{A}_{0}=\left[\begin{array}{cc}
\frac{1}{2} \mathbf{I} & \mathbf{0} \\
\mathbf{0} & \mathbf{0}
\end{array}\right] \\
& \mathbf{A}_{1}=\left[\begin{array}{cc}
\frac{1}{2}\left(\mathbf{I}+\frac{1}{k}\left(\mathbf{T}_{11}-\delta \mathbf{I}\right)\right) & \mathbf{0} \\
\frac{1}{k} \mathbf{T}_{21, c} & \mathbf{0}
\end{array}\right]
\end{aligned}
$$

and

is of the form

$$
\mathbf{A}_{2}=\left[\begin{array}{cc}
\mathbf{0} & \frac{1}{2 k} \mathbf{T}_{12} \\
\mathbf{0} & \left(\mathbf{I}+\frac{1}{k}\left(\mathbf{T}_{22, c}-\delta \mathbf{I}\right)\right)
\end{array}\right]
$$

where

$$
\mathbf{Y}=\left[\begin{array}{cc}
\mathbf{0} & \boldsymbol{\Psi}_{c}(\delta) \\
\mathbf{0} & \mathbf{I}+\frac{1}{k} \mathbf{H}_{c}(\delta)
\end{array}\right],
$$

$$
\mathbf{H}_{c}(\delta)=c^{-1}\left[\mathbf{T}_{22, c}-\delta \mathbf{I}+\mathbf{T}_{21, c} \boldsymbol{\Psi}_{c}(\delta)\right]
$$

Note that the constant $k$ in the matrices $\mathbf{A}_{0}, \mathbf{A}_{1}, \mathbf{A}_{2}$ and $\mathbf{Y}$ shall be greater than the largest absolute value among the diagonal elements of the matrix $\mathbf{T}-\delta \mathbf{I}$.

Thus, it suffices to solve (3) or (4) in order to compute $\boldsymbol{\Psi}_{c}(\delta)$ for any $\delta \geq 0$. Essentially, two main families of approaches are available to tackle this problem: spectral decomposition and iterative schemes. The procedures developed in e.g. Asmussen [10, (1995)] and Asmussen et al. [12, (2002)] when the arrival process forms a renewal process belong to the first family. From our experience, spectral decomposition and root finding methods tend to be more susceptible to numerical problem/instability (especially when the number of roots become large) in comparison with iterative schemes. The Log-Reduction (LR) algorithm of Latouche and Ramaswami [43, (1993)] for the computation of $\boldsymbol{\Psi}_{c}(\delta)$ is an example of iterative scheme. The LR algorithm is designed to reduce the number of unnecessary iterations needed to obtain the solution of (4). The algorithm works only for quasi-birth-and-death-processes (QBDs) that are the discrete equivalent of the fluid flows presented in this section. For the sake of completeness, we review the LR algorithm in appendix A. A few years later, Ahn and Ramaswami [4, (2005)] provided an improved algorithm for computing this first passage probability matrix and demonstrated that it converges quadratically fast, yielding very accurate numerical results.

In the following subsection, additional LSTs of first passage times are discussed in the fluid flow $\underline{F_{c}}$. The LST of a busy period $\Psi_{c}(\delta)$ will play a predominant role in most of these newly defined LSTs. 


\subsubsection{Other first passage probabilities}

In Ahn and Ramaswami [3, 5, (2004, 2006)], the matrices $\mathbf{H}_{c}(\delta)$ and $\mathbf{K}_{c}(\delta)$ are introduced where $\mathbf{H}_{c}(\delta)$ is as defined in (5) while

$$
\mathbf{K}_{c}(\delta)=c^{-1}\left[\mathbf{T}_{11}-\delta \mathbf{I}+\mathbf{\Psi}_{c}(\delta) \mathbf{T}_{21, c}\right] .
$$

Both $\mathbf{H}_{c}(\delta)$ and $\mathbf{K}_{c}(\delta)$ have nice probabilistic interpretations. For $x, y \geq 0$, $\left[\mathrm{e}^{\mathbf{H}_{c}(\delta) x}\right]_{i j}$ represents the LST of the first passage time of the fluid flow $\left(\underline{F_{c}}, \underline{W}\right)$ from $\left(x+y, i \in S_{2}\right)$ to $\left(y, j \in S_{2}\right)$, whereas $\left[\mathrm{e}^{\mathbf{K}_{c}(\delta) x}\right]_{i j}$ is the LST of the expected number of crossings of the fluid flow $\left(\underline{F_{c}}, \underline{W}\right)$ to level $x+y$, phase $j \in S_{1}$ while avoiding level $y$ enroute, given that the process started in $\left(y, i \in \overline{S_{1}}\right)$. Noting that the last quantity of interest is the LST of a certain Markov renewal kernel, it can also be interpreted as the Laplace transform of the density associated with the relevant taboo crossing (see Ahn and Ramaswami [3, 5, (2004, 2006)] for a detailed explanation).

In addition, Ramaswami [55, (2006)] defined yet another matrix, namely

$$
\mathbf{U}_{c}(\delta, x)=\int_{0}^{x} \mathrm{e}^{\mathbf{H}_{c}(\delta) y} c^{-1} \mathbf{T}_{21, c} \mathrm{e}^{\mathbf{K}_{c}(\delta) y} \mathrm{~d} y,
$$

where $\left[\mathbf{U}_{c}(\delta, x)\right]_{i j}$ represents the LST of the expected number of crossings of the fluid flow $\left(F_{c}, \underline{W}\right)$ to level $x$, phase $j \in S_{1}$ while avoiding level 0 enroute given that the process started in $\left(x, i \in S_{2}\right)$. Note that the integral in (6) can be calculated exactly using Lemma 2 in Ramaswami [55, (2006)].

Analogous to the above matrices pertaining to the fluid flow $\left(\underline{F_{c}}, \underline{W}\right)$, we can also introduce the matrices $\mathbf{\Psi}_{c}^{r}(\delta), \mathbf{H}_{c}^{r}(\delta), \mathbf{K}_{c}^{r}(\delta)$, and $\mathbf{U}_{c}^{r}(\delta, x)$ which pertain to the reflected fluid flow $\left(F_{c}^{r}, \underline{W}\right)$. Their formulae and probabilistic interpretations are similar to the above-mentioned ones and can also be found in Ahn et al. [2, (2007)]. An important aspect that is common to all of the above quantities is the fact that their calculation essentially reduces to the evaluation of the first passage probability matrix $\boldsymbol{\Psi}_{c}(\delta)$, whose evaluation has already been discussed in Section 2.3.1. We are now ready to review some other useful LSTs of first passage times.

For $i, j=1,2$, we define the $\left|S_{i}\right| \times\left|S_{j}\right|$ matrix of the LST of the first passage time of the fluid flow process $\left(\underline{F_{c}}, \underline{J}\right)$ from $\left(x, S_{i}\right)$ to $\left(y, S_{j}\right)$ while avoiding a visit to the levels in $[0, a]$ and $[z, \infty)$ enroute, i.e.

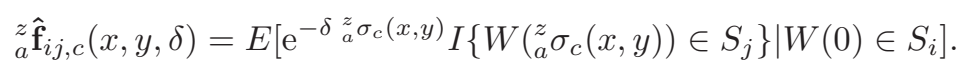

We remark that the superscript " $r$ " will be added to the above quantity in the reflected version of the fluid flow $\left(\underline{F_{c}}, \underline{W}\right)$. The same comment with respect to the suppression of the arguments $a$ and $z$ that we have made earlier applies here as well.

Ramaswami [55, (2006)] obtained formulae for many important first passage times in infinite buffer fluid flow models. In Table 1, we list the pertinent results. The two formulae stated in Table 1 without reference immediately follow from equivalent results pertaining to the fluid flow $\underline{F_{c}}$.

We end this section by stating (without proof) several other useful LSTs derived in Theorem 1 of Ahn et al. [2, (2007)]. Indeed, for $0 \leq x<y$,

$$
\begin{aligned}
{ }^{y} \hat{\mathbf{f}}_{22, c}(x, 0, \delta) & =\left[\mathbf{I}-{ }^{x} \mathbf{\Psi}_{c}^{r}(\delta) \cdot{ }^{y-x} \mathbf{\Psi}_{c}(\delta)\right]^{-1} \cdot{ }^{x} \hat{\mathbf{f}}_{22, c}(x, 0, \delta), \\
{ }^{y} \hat{\mathbf{f}}_{12, c}(x, 0, \delta) & ={ }^{y-x} \boldsymbol{\Psi}_{c}(\delta) \cdot{ }^{y} \hat{\mathbf{f}}_{22, c}(x, 0, \delta), \\
{ }_{0} \hat{\mathbf{f}}_{11, c}(x, y, \delta) & =\left[\mathbf{I}-{ }^{y-x} \mathbf{\Psi}_{c}(\delta) \cdot{ }^{x} \mathbf{\Psi}_{c}^{r}(\delta)\right]^{-1} \cdot{ }_{0} \hat{\mathbf{f}}_{11, c}(0, y-x, \delta), \\
{ }_{0} \hat{\mathbf{f}}_{21, c}(x, y, \delta) & ={ }^{x} \boldsymbol{\Psi}_{c}^{r}(\delta) \cdot{ }_{0} \hat{\mathbf{f}}_{11, c}(x, y, \delta) .
\end{aligned}
$$

\section{Applications of MAMs in ruin theory}

In this section, we consider the applications of MAMs in the context of two risk processes: the surplus process $\underline{R}$ described in Section 1.3 and its modified version in the presence of a threshold dividend strategy 


\begin{tabular}{|c|c|c|c|}
\hline LST & First passage time & Formula & Ref. \\
\hline$\hat{\mathbf{f}}_{22, c}(x, 0, \delta)$ & $\begin{array}{l}\text { from }\left(x, S_{2}\right) \text { to }\left(0, S_{2}\right) \\
\text { in } \underline{F_{c}}\end{array}$ & $\mathrm{e}^{\mathbf{H}_{c}(\delta) x}$ & $\begin{array}{c}\text { Ahn and Ramaswami } \\
{[4,(2005)], \text { Th. } 3}\end{array}$ \\
\hline$\hat{\mathbf{f}}_{11, c}^{r}(x, 0, \delta)$ & $\begin{array}{l}\text { from }\left(x, S_{1}\right) \text { to }\left(0, S_{1}\right) \\
\text { in } F_{c}{ }^{r}\end{array}$ & $\mathrm{e}^{\mathbf{H}_{c}^{r}(\delta) x}$ & \\
\hline${ }_{0} \hat{\mathbf{f}}_{11, c}(0, x, \delta)$ & $\begin{array}{l}\text { from }\left(0, S_{1}\right) \text { to }\left(x, S_{1}\right) \\
\text { avoiding } 0 \text { enroute in } \underline{F_{c}}\end{array}$ & $\mathrm{e}^{\mathbf{K}_{c}(\delta) x}\left[\mathbf{I}_{1}+\mathbf{\Psi}_{c}(\delta) \mathbf{U}_{c}(\delta, x)\right]^{-1}$ & $\begin{array}{c}\text { Ramaswami } \\
{[55,(2006)], \text { Th. } 1}\end{array}$ \\
\hline${ }_{0} \hat{\mathbf{f}}_{22, c}^{r}(0, x, \delta)$ & $\begin{array}{l}\text { from }\left(0, S_{2}\right) \text { to }\left(x, S_{2}\right) \\
\text { avoiding } 0 \text { enroute in }{\underline{F_{c}}}^{r}\end{array}$ & $\mathrm{e}^{\mathbf{K}_{c}^{r}(\delta) x}\left[\mathbf{I}_{1}+\mathbf{\Psi}_{c}^{r}(\delta) \mathbf{U}_{c}^{r}(\delta, x)\right]^{-1}$ & \\
\hline $\begin{array}{c}{ }^{x} \boldsymbol{\Psi}_{c}(\delta)= \\
{ }^{x} \hat{\mathbf{f}}_{12, c}(0,0, \delta)\end{array}$ & $\begin{array}{l}\text { from }\left(0, S_{1}\right) \text { to }\left(0, S_{2}\right) \\
\text { avoiding } x \text { enroute in } F_{c}\end{array}$ & $\boldsymbol{\Psi}_{c}(\delta)-{ }_{0} \hat{\mathbf{f}}_{11, c}(0, x, \delta) \boldsymbol{\Psi}_{c}(\delta) \mathrm{e}^{\mathbf{H}_{c}(\delta) x}$ & $\begin{array}{c}\text { Ramaswami } \\
{[55,(2006)], \text { Th. } 2}\end{array}$ \\
\hline${ }^{x} \hat{\mathbf{f}}_{22, c}(x, 0, \delta)$ & $\begin{array}{l}\text { from }\left(x, S_{2}\right) \text { to }\left(0, \overline{S_{2}}\right) \\
\text { avoiding } x \text { enroute in } \underline{F_{c}}\end{array}$ & ${ }_{0} \hat{\mathbf{f}}_{22, c}^{r}(0, x, \delta)$ & $\begin{array}{c}\text { Ramaswami } \\
{[55,(2006)], \text { Th. } 3}\end{array}$ \\
\hline $\begin{array}{c}{ }^{x} \mathbf{\Psi}_{c}^{r}(\delta)= \\
{ }_{0} \hat{\mathbf{f}}_{21, c}(x, x, \delta)\end{array}$ & $\begin{array}{l}\text { from }\left(0, S_{2}\right) \text { to }\left(0, S_{1}\right) \\
\text { avoiding } x \text { enroute in } \underline{F_{c}}\end{array}$ & $\mathbf{\Psi}_{c}^{r}(\delta)-{ }_{0} \hat{\mathbf{f}}_{22, c}^{r}(0, x, \delta) \boldsymbol{\Psi}_{c}^{r}(\delta) \mathrm{e}^{\mathbf{H}_{c}^{r}(\delta) x}$ & $\begin{array}{c}\text { Ramaswami } \\
{[55,(2006)], \text { Th. } 4}\end{array}$ \\
\hline
\end{tabular}

Table 1. First passage time results in fluid flow models

(see Section 3.3 below). We stress that these are only two illustrative examples of MAMs applications in ruin theory and by no means represent the full extent of MAMs applications for the study of surplus processes. The reader is referred to Section 4 for further applications of MAMs in frequently analyzed ruin theory problems. But first, a brief review of some popular ruin-related quantities in ruin theory is presented.

\subsection{Ruin-related quantities of interest}

For many years, ruin probabilities and many ruin-related quantities such as the marginal and joint defective distributions of the deficit at ruin, the surplus just prior to ruin and the claim size causing ruin have been extensively studied in various risk models (see e.g. Dufresne and Gerber [38, (1988)], Dickson [35, 36, (1992, 1993)], Gerber and Shiu [39, (1997)], Dickson and Hipp [37, (1998)] and references therein). For a given surplus process, a common ground seems to exists in the study of a number of these ruin-related quantities. This idea was brilliantly formalized in Gerber and Shiu's [40, (1998)] seminal paper where the so-called expected discounted penalty function was proposed. For a surplus process $\underline{R}$, the Gerber-Shiu discounted penalty function is defined as

$$
\phi_{\delta}(u)=E\left[\mathrm{e}^{-\delta \tau} w\left(R\left(\tau^{-}\right),|R(\tau)|\right) I(\tau<\infty) \mid R(0)=u\right],
$$

where $\tau=\inf \{t \geq 0: R(t)<0\}$ is the time of ruin (with $\tau=\infty$ if ruin does not occur), $R\left(\tau^{-}\right.$) is the surplus immediately before ruin, $|R(\tau)|$ is the deficit at ruin, and $w: \mathbb{R}^{2} \rightarrow \mathbb{R}$ is a penalty function which is assumed to satisfy some mild integrability conditions.

The strenght of the new analytic tool (11) resides in the fact that it generalizes a number of commonly analyzed ruin-related quantities (see those mentioned above) in addition of providing a platform for the study of new ones via a proper choice of the penalty function $w$. Some examples are provided here:

1. for $w(x, y)=1$ for all $x, y \geq 0, \phi_{\delta}(u)$ corresponds to the Laplace transform of the time to ruin. When $\delta=0, \phi_{0}(u)$ corresponds to the probability of ruin.

2. for $w(x, y)=\Delta(x-v) \Delta(y-z)$ for all $x, y \geq 0$ (where $\Delta$ is the Dirac delta function), $\phi_{\delta}(u)$ corresponds to the so-called discounted density of $\left(R\left(\tau^{-}\right),|R(\tau)|\right)$ at $(v, z)$. When $\delta=0, \phi_{0}(u)$ corresponds to the (defective) density of $\left(R\left(\tau^{-}\right),|R(\tau)|\right)$ at $(v, z)$.

3. for $w(x, y)=y^{k}$ for all $x, y \geq 0$ and $\delta=0, \phi_{\delta}(u)$ corresponds to the $k$-th moment of the deficit at ruin. 
Moreover, quantities such as the joint moments of the time of ruin, the surplus prior to ruin and the deficit at ruin, as well as their associated marginal moments and the distribution of the claim causing ruin can easily be derived via a Gerber-Shiu type analysis. A detailed analysis of some of these quantities in the classical compound Poisson risk model can be found in e.g. Lin and Willmot [48, (1999)].

Due to its flexibility and unifying properties, several authors have since then characterized Gerber-Shiu's discounted penalty function under different risk model assumptions. We mention Gerber and Shiu [41, (2005)] and Li and Garrido [45, (2005)] in the context of the Sparre Andersen risk model, Lu and Li [49, (2009)] and Lu and Tsai [50, (2007)] in the Markov-modulated risk model, and Cheung and Landriault [32, (2009)] in the MAP risk model, among others.

For the MAP risk model, the Gerber-Shiu discounted penalty function is defined conditional on the state of the underlying Markovian environmental process at time 0 . Hence, let

$$
\phi_{\delta, i}(u)=E\left[\mathrm{e}^{-\delta \tau} w\left(R\left(\tau^{-}\right),|R(\tau)|\right) I(\tau<\infty) \mid R(0)=u, J(0)=i\right],
$$

be the Gerber-Shiu function with $J(0)=i\left(i \in S_{1}\right)$.

As pointed out by Gerber and Shiu [39, (1997)], Wu et al. [60, (2003)] and Landriault and Willmot [42, (2009)], the joint density of $\tau, R\left(\tau^{-}\right)$and $|R(\tau)|$ takes different forms depending on whether ruin occurs on the first claim (i.e. $N(\tau)=1$ ) or on any subsequent claim to the first (i.e. $N(\tau)>1$ ). For ruin occurring on the first claim, let $h_{1, i}(x, y \mid u)$ be the joint density of $\left(R\left(\tau^{-}\right),|R(\tau)|\right)$ at $(x, y)$ given that $J(0)=i$ and $R(0)=u$. In this case, it is clear that $\tau=(x-u) / c$. If ruin occurs on claims subsequent to the first, we denote the joint density of $\left(\tau, R\left(\tau^{-}\right),|R(\tau)|\right)$ at $(t, x, y)$ given that $J(0)=i$ and $R(0)=u$ by $h_{2, i}(t, x, y \mid u)$ for $t, x, y>0$.

By conditioning on the time of ruin $\tau$, the surplus immediately prior to ruin $R\left(\tau^{-}\right)$and the deficit at ruin $|R(\tau)|$, one obtains

$$
\begin{aligned}
\phi_{\delta, i}(u)= & \int_{u}^{\infty} \int_{0}^{\infty} \mathrm{e}^{-\delta\left(\frac{x-u}{c}\right)} w(x, y) h_{1, i}(x, y \mid u) \mathrm{d} y \mathrm{~d} x \\
& +\int_{0}^{\infty} \int_{0}^{\infty} \int_{0}^{\infty} \mathrm{e}^{-\delta t} w(x, y) h_{2, i}(t, x, y \mid u) \mathrm{d} y \mathrm{~d} x \mathrm{~d} t .
\end{aligned}
$$

Letting

$$
g_{u, i}(\delta, x, y)=\left\{\begin{array}{ll}
\mathrm{e}^{-\delta\left(\frac{x-u}{c}\right)} h_{1, i}(x, y \mid u)+\int_{0}^{\infty} \mathrm{e}^{-\delta t} h_{2, i}(t, x, y \mid u) \mathrm{d} t, & x>u \\
\int_{0}^{\infty} \mathrm{e}^{-\delta t} h_{2, i}(t, x, y \mid u) \mathrm{d} t, & 0<x<u
\end{array},\right.
$$

for $y>0$ be the discounted joint density of the surplus prior to ruin $(x)$ and the deficit at ruin $(y)$, it allows to rewrite (12) as

$$
\phi_{\delta, i}(u)=\int_{0}^{\infty} \int_{0}^{\infty} w(x, y) g_{u, i}(\delta, x, y) \mathrm{d} y \mathrm{~d} x .
$$

For convenience, we also define the following two column vectors: $\phi_{\delta}(u)=\left[\phi_{\delta, i}(u)\right]_{i=1}^{m}$ and $\mathbf{g}_{u}(\delta, x, y)=$ $\left[g_{u, i}(\delta, x, y)\right]_{i=1}^{m}$.

Note that the discounted joint density $\mathbf{g}_{u}(\delta, x, y)$ does not depend on the penalty function $w$. Hence, one can first identify $\mathbf{g}_{u}(\delta, x, y)$. From the knowledge of $\mathbf{g}_{u}(\delta, x, y)$ together with (13), calculations of GerberShiu functions for different choices of penalty function $w$ immediately follows. Thus, in the following two subsections, we identify $\mathbf{g}_{u}(\delta, x, y)$ for two risk processes.

We remark that the discounted joint density of the surplus prior to ruin and the deficit at ruin has been studied by e.g. Gerber and Shiu [39, (1997)], Li and Garrido [45, (2005)] and Ren [57, (2007)]. A generalized version of this discounted joint density can also be found in Breuer [30, (2009)] and Cheung et al. [33, (2009)]. 


\subsection{The basic insurance risk model}

Using the LST of some first passage times in the MMFF $\left(\underline{F_{c}}, \underline{W}\right)$, we identify the discounted density of the surplus prior to ruin $(x)$ and the deficit at ruin $(y)$ for the surplus process $\underline{R}$ with time to ruin $\tau \equiv \tau_{c}(u, 0)$ (see Section 2.2). The reader is referred to Badescu et al. [21, (2005)] for an expression for the nondiscounted (i.e. $\delta=0$ ) version of this joint density. Theorem 2 has been first proved by Ramaswami [55, (2006)].

Theorem 2 The discounted joint density of the surplus prior to ruin $(x)$ and the deficit at ruin $(y)$, $\mathbf{g}_{u}(\delta, x, y)$, is given by:

- for $0<u<x$ and $y \geq 0$,

$$
\mathbf{g}_{u}(\delta, x, y)=\mathrm{e}^{\frac{-\delta(x-u)}{2 c}}{ }_{0} \hat{\mathbf{f}}_{11, c}(u, x, \delta / 2)\left[\mathbf{I}-\mathbf{\Psi}_{c}(\delta / 2)^{x} \mathbf{\Psi}_{c}^{r}(\delta / 2)\right]^{-1}\left(c^{-1} \mathbf{T}_{12}\right) \mathrm{e}^{\mathbf{T}_{22,1}(x+y)} \mathbf{t}_{21,1} \cdot
$$

- for $0<x<u$ and $y \geq 0$,

$$
\begin{aligned}
\mathbf{g}_{u}(\delta, x, y)=\mathrm{e}^{\frac{\delta(u-x)}{2 c}} \hat{\mathbf{f}}_{12, c}(u-x, 0, \delta / 2)^{x} \boldsymbol{\Psi}_{c}^{r}(\delta / 2)\left[\mathbf{I}-\boldsymbol{\Psi}_{c}(\delta / 2)^{x} \boldsymbol{\Psi}_{c}^{r}(\delta / 2)\right]^{-1} \\
\quad \times\left(c^{-1} \mathbf{T}_{12}\right) \mathrm{e}^{\mathbf{T}_{22,1}(x+y)} \mathbf{t}_{21,1} .
\end{aligned}
$$

Proof. For an initial surplus $u<x$, a contribution to $\mathbf{g}_{u}(\delta, x, y)$ is made only if the surplus process $\underline{R}$ reaches level $x$ at least once prior to ruin. Using Remark 1 , the LST of this first passage time is $\mathrm{e}^{-\frac{\delta}{2 c}(x-y)}{ }_{0} \hat{\mathbf{f}}_{11, c}(u, x, \delta / 2)$. Now at level $x$ in an increasing phase, the surplus process can re-visit level $x$ in an increasing phase an arbitrary number of times prior to ruin. The LST of this duration of time is given by $\left[\mathbf{I}-\boldsymbol{\Psi}_{c}(\delta / 2)^{x} \boldsymbol{\Psi}_{c}^{r}(\delta / 2)\right]^{-1}$. Finally, from level $x$ in an increasing phase, the surplus process shall experience a claim in the next $c^{-1} \mathrm{~d} t$ time interval (with contribution $\mathbf{T}_{21,1}$ ) of size $x+y$ to enable a deficit at ruin of $y$ (with contribution $\left.\exp \left\{\mathbf{T}_{22,1}(x+y)\right\} \mathbf{t}_{21,1}\right)$.

Let us now prove (14). For an initial surplus $u>x$, the equivalent fluid flow process has to make a first passage to level $x$ in a decreasing phase. Using Remark 1, the LST of this first passage time in the surplus process $\underline{R}$ is given by $\mathrm{e}^{\frac{\delta}{2 c}(u-x)} \hat{\mathbf{f}}_{12, c}(u-x, 0, \delta / 2)$. Now at level $x$ (with a phase in $S_{2}$ ) for the first time, it is possible to re-visit level $x$ (with a phase in $S_{1}$ ) an arbitrary number of times prior to ruin. The LST of this duration of time in the corresponding risk model is given by ${ }^{x} \boldsymbol{\Psi}_{c}^{r}(\delta / 2) \cdot\left[\mathbf{I}-\boldsymbol{\Psi}_{c}(\delta / 2) \cdot{ }^{x} \mathbf{\Psi}_{c}^{r}(\delta / 2)\right]^{-1}$. Finally, as in the proof above, the equivalent fluid flow must switch immediately from an increasing phase to a decreasing phase followed by a period of descent (at rate $c$ ) of length $(x+y) / c$.

Furthermore, one can derive the LST of the time to ruin $\tau$ for the surplus process $\underline{R}$ via a double integration of $\mathbf{g}_{u}(\delta, x, y)$, i.e.

$$
\begin{aligned}
\rho_{u}(\delta) & \equiv E\left[\mathrm{e}^{-\delta \tau} 1(\tau<\infty) \mid R(0)=u\right] \\
& =\int_{0}^{\infty} \int_{0}^{\infty} \mathbf{g}_{u}(\delta, x, y) \mathrm{d} y \mathrm{~d} x
\end{aligned}
$$

Analyses of the Laplace transform $\rho_{u}(\delta)$ were conducted by Badescu et al. [20, (2005)] and Ramaswami [55, (2006)]. For purposes of completeness, Ramaswami [55, (2006)] has shown that

$$
\rho_{u}(\delta)=\Psi_{c}(\delta / 2) \mathrm{e}^{\frac{\delta}{2} \frac{u}{c}} \hat{\mathbf{f}}_{12, c}(u, 0, \delta / 2) .
$$

Finally, we refer the reader to Ahn and Badescu [1, (2007)] for a more complete discussion on the study of Gerber-Shiu functions in the present risk process. 


\subsection{The insurance risk model with a threshold strategy}

In recent years, risk processes modified by various dividend payment strategies have received considerable attention in the actuarial literature on ruin theory. Risk models with a dividend threshold strategy have been studied by e.g. Lin and Pavlova [46, (2005)] in the classical compound Poisson risk model, Albrecher et al. [8, (2006)] in the Sparre Andersen risk model and Lu and Li [49, (2009)] in the Markov-modulated risk model. The surplus process in the presence of a threshold strategy at level $b$, namely $\left\{R^{b}(t), t \geq 0\right\}$, is defined as

$$
\mathrm{d} R^{b}(t)=\left\{\begin{array}{ll}
c_{1} \mathrm{~d} t-\mathrm{d}\left(\sum_{k=1}^{N(t)} X_{k}\right), & R^{b}(t)<b \\
c_{2} \mathrm{~d} t-\mathrm{d}\left(\sum_{k=1}^{N(t)} X_{k}\right), & R^{b}(t) \geq b
\end{array},\right.
$$

where all the r.v's in (15) are as defined in Section 1.3. Typically, the premium rates $c_{1}$ and $c_{2}$ satisfies the inequality $c_{1} \geq c_{2}$ with $c_{1}-c_{2}$ being paid as a dividend to the shareholders whenever the surplus level lies above the threshold level $b$.

Let $\tau^{b}$ be the time to ruin for the surplus process $\left\{R^{b}(t), t \geq 0\right\}$. An explicit form for the discounted density of the surplus prior to ruin $R^{b}\left(\tau^{b}-\right)$ and the deficit at ruin $\left|R^{b}\left(\tau^{b}\right)\right|$ is given below. To this end, let $\mathbf{g}_{u}^{b}(\delta, x, y)$ be a $\left|S_{1}\right| \times 1$ column vector for which its $i$-th element $\left[\mathbf{g}_{u}^{b}(\delta, x, y)\right]_{i}$ is defined as

$$
\left[\mathbf{g}_{u}^{b}(\delta, x, y)\right]_{i} \mathrm{~d} x \mathrm{~d} y=E\left[\mathrm{e}^{-\delta \tau^{b}} I\left\{R^{b}\left(\tau^{b}-\right) \in(x, x+d x)\right\} I\left\{\left|R^{b}\left(\tau^{b}\right)\right| \in(y, y+d y)\right\} \mid J(0)=i\right] .
$$

Several lemmas are first introduced to define additional LSTs of first passage times in a particular fluid flow process.

In what follows, we consider a fluid flow that accumulates/depletes at rate $c_{1}$ for levels between 0 and $x$ and at rate $c_{2}$ for levels above $x$. We define ${ }_{a}^{z} \hat{\mathbf{f}}_{i j, c_{1}, c_{2}}(x, y, \cdot), i, j=1,2$, to be the $\left|S_{i}\right| \times\left|S_{j}\right|$ matrix of the LST of the first passage time of this fluid flow process from $\left(x, S_{i}\right)$ to $\left(y, S_{j}\right)$ while avoiding a visit to the levels in $[0, a]$ and $[z, \infty)$ enroute. The following first passage time LSTs, analogous to the ones given in (7), (8), (9), and (10), are presented in the following lemma.

Lemma 1 For $0 \leq x<y$,

$$
\begin{aligned}
{ }^{y} \hat{\mathbf{f}}_{22, c_{1}, c_{2}}(x, 0, \delta) & =\left[\mathbf{I}-{ }^{x} \boldsymbol{\Psi}_{c_{1}}^{r}(\delta) \cdot{ }^{y-x} \boldsymbol{\Psi}_{c_{2}}(\delta)\right]^{-1} \cdot{ }^{x} \hat{\mathbf{f}}_{22, c_{1}}(x, 0, \delta), \\
{ }^{y} \hat{\mathbf{f}}_{12, c_{1}, c_{2}}(x, 0, \delta) & ={ }^{y-x} \boldsymbol{\Psi}_{c_{2}}(\delta) \cdot{ }^{y} \hat{\mathbf{f}}_{22, c_{1}, c_{2}}(x, 0, \delta), \\
{ }_{0} \hat{\mathbf{f}}_{11, c_{1}, c_{2}}(x, y, \delta) & =\left[\mathbf{I}-{ }^{y-x} \mathbf{\Psi}_{c_{2}}(\delta) \cdot{ }^{x} \mathbf{\Psi}_{c_{1}}^{r}(\delta)\right]^{-1} \cdot{ }_{0} \hat{\mathbf{f}}_{11, c_{2}}(0, y-x, \delta), \\
{ }_{0} \hat{\mathbf{f}}_{21, c_{1}, c_{2}}(x, y, \delta) & ={ }^{x} \mathbf{\Psi}_{c_{1}}^{r}(\delta) \cdot{ }_{0} \hat{\mathbf{f}}_{11, c_{1}, c_{2}}(x, y, \delta) .
\end{aligned}
$$

ProOF. Starting at level $x$ (with a phase in $S_{2}$ ), it is possible for the fluid flow process to make several visits back to level $x$ while avoiding levels 0 and $y$ enroute. Therefore, the LST of the time until the last visit to $\left(x, S_{2}\right)$ given that the process started in $\left(x, S_{2}\right)$ is given by $\left[\mathbf{I}-{ }^{x} \boldsymbol{\Psi}_{c_{1}}^{r}(\delta)^{y-x} \boldsymbol{\Psi}_{c_{2}}(\delta)\right]^{-1}$. Now at level $x$ (with a phase in $S_{2}$ ) for the last time, the fluid flow process must make a first passage to $\left(0, S_{2}\right)$, and this LST is given by ${ }^{x} \hat{\mathbf{f}}_{22, c_{1}}(x, 0, \delta)$. This completes the proof of (16). Similar arguments lead to the expressions in (17), (18) and (19).

Letting $y=\infty$ in (16) and (17), one obtains the following identities:

Corollary 1 For $0 \leq x<\infty$,

$$
\begin{aligned}
& \hat{\mathbf{f}}_{22, c_{1}, c_{2}}(x, 0, \delta)=\left[\mathbf{I}-{ }^{x} \boldsymbol{\Psi}_{c_{1}}^{r}(\delta) \cdot \boldsymbol{\Psi}_{c_{2}}(\delta)\right]^{-1} \cdot{ }^{x} \hat{\mathbf{f}}_{22, c_{1}}(x, 0, \delta), \\
& \hat{\mathbf{f}}_{12, c_{1}, c_{2}}(x, 0, \delta)=\boldsymbol{\Psi}_{c_{2}}(\delta) \cdot \hat{\mathbf{f}}_{22, c_{1}, c_{2}}(x, 0, \delta) .
\end{aligned}
$$

Lemma 1 and Corollary 1 provide the basic tools necessary to construct the following first passage time LSTs that are essential in the derivation of the main result of this section. The proofs of the following lemmas/corollaries can be found in Badescu et al. [23, (2007)]. 
Lemma 2 Consider a fluid flow process which accumulates/depletes at rate $c_{1}$ for levels in $(0, x)$ and at rate $c_{2}$ for levels in $[x, \infty)$. For $0<x<y$, the $\left|S_{1}\right| \times\left|S_{2}\right|$ matrix of the LST of the return time of this fluid flow from $\left(0, S_{1}\right)$ to $\left(0, S_{2}\right)$ avoiding level $y$ enroute is given by

$$
{ }^{y} \Psi_{x, c_{1}, c_{2}}(\delta)={ }^{x} \boldsymbol{\Psi}_{c_{1}}(\delta)+{ }_{0} \hat{\mathbf{f}}_{11, c_{1}}(0, x, \delta){ }^{y} \hat{\mathbf{f}}_{12, c_{1}, c_{2}}(x, 0, \delta) .
$$

The previous result can be further simplified when $y \rightarrow \infty$.

Corollary 2 Consider the fluid flow described in Lemma 2 with $y=\infty$. The $\left|S_{1}\right| \times\left|S_{2}\right|$ matrix of the LST of the return time of the fluid flow from $\left(0, S_{1}\right)$ to $\left(0, S_{2}\right)$ is given by

$$
\boldsymbol{\Psi}_{x, c_{1}, c_{2}}(\delta)={ }^{x} \boldsymbol{\Psi}_{c_{1}}(\delta)+{ }_{0} \hat{\mathbf{f}}_{11, c_{1}}(0, x, \delta) \cdot \hat{\mathbf{f}}_{12, c_{1}, c_{2}}(x, 0, \delta) .
$$

The last preliminary result concerns the LST of the first passage time from $\left(0, S_{2}\right)$ to $\left(0, S_{1}\right)$ in the reflected version of the above fluid flow model.

Lemma 3 Consider the reflected version of the fluid flow described in Lemma 2 which accumulates/depletes at rate $c_{2}$ for levels in the interval $(0, x)$ and at rate $c_{1}$ for levels in the interval $[x, \infty)$. For $0<x<y$, the $\left|S_{2}\right| \times\left|S_{1}\right|$ matrix of the LST of the return time of the reflected fluid flow from $\left(0, S_{2}\right)$ to $\left(0, S_{1}\right)$ avoiding level y enroute is given by ${ }^{y} \mathbf{\Psi}_{x, c_{2}, c_{1}}^{r}(\delta)={ }^{x} \boldsymbol{\Psi}_{c_{2}}^{r}(\delta)+{ }^{x} \hat{\mathbf{f}}_{22, c_{2}}(x, 0, \delta) \cdot{ }_{0} \hat{\mathbf{f}}_{21, c_{1}, c_{2}}(y-x, y, \delta)$.

The main result of this section is given in the next theorem which is due to Badescu et al. [23, (2007)]. We refer to the original paper for a proof of this result.

Theorem 3 The joint discounted density of the surplus prior to ruin $(x)$ and the deficit at ruin $(y)$, $\mathbf{g}_{u}^{b}(\delta, x, y)$, in the surplus process $\left\{R^{b}(t), t \geq 0\right\}$ is given by:

1 for $u \leq b$,

(a) for $b<x$ and $y>0$,

$$
\begin{aligned}
\mathbf{g}_{u}^{b}(\delta, x, y)=c_{2}^{-1} \mathrm{e}^{\frac{-\delta(b-u)}{2 c_{1}}} \mathrm{e}^{\frac{-\delta(x-b)}{2 c_{2}}}{ }_{0} \hat{\mathbf{f}}_{11, c_{1}}(u, b, \delta / 2) \cdot{ }_{0} \hat{\mathbf{f}}_{11, c_{1}, c_{2}}(b, x, \delta / 2) \\
\cdot\left[\mathbf{I}-\boldsymbol{\Psi}_{c_{2}}(\delta / 2) \cdot{ }^{x} \mathbf{\Psi}_{x-b, c_{2}, c_{1}}^{r}(\delta / 2)\right]^{-1} \cdot \mathbf{T}_{12} \mathrm{e}^{\mathbf{T}_{22,1}(x+y)} \mathbf{t}_{21,1}
\end{aligned}
$$

(b) for $u<x<b$ and $y>0$,

$$
\begin{gathered}
\mathbf{g}_{u}^{b}(\delta, x, y)=c_{1}^{-1} \mathrm{e}^{\frac{-\delta(x-u)}{2 c_{1}}}{ }_{0} \hat{\mathbf{f}}_{11, c_{1}}(u, x, \delta / 2)\left[\mathbf{I}-\mathbf{\Psi}_{b-x, c_{1}, c_{2}}(\delta / 2) \cdot{ }^{x} \mathbf{\Psi}_{c_{1}}^{r}(\delta / 2)\right]^{-1} \\
\cdot \mathbf{T}_{12} \mathrm{e}^{\mathbf{T}_{22,1}(x+y)} \mathbf{t}_{21,1} .
\end{gathered}
$$

(c) for $0<x<u$ and $y>0$,

$$
\begin{gathered}
\mathbf{g}_{u}^{b}(\delta, x, y)=c_{1}^{-1} \rho_{\delta}^{b-x}(u-x) \cdot{ }^{x} \mathbf{\Psi}_{c_{1}}^{r}(\delta / 2)\left[\mathbf{I}-\mathbf{\Psi}_{b-x, c_{1}, c_{2}}(\delta / 2) \cdot{ }^{x} \boldsymbol{\Psi}_{c_{1}}^{r}(\delta / 2)\right]^{-1} \\
\cdot \mathbf{T}_{12} \mathrm{e}^{\mathbf{T}_{22,1}(x+y)} \mathbf{t}_{21,1} .
\end{gathered}
$$

2 for $0<b<u$,

(a) for $u<x$ and $y>0$,

$$
\begin{aligned}
\mathbf{g}_{u}^{b}(\delta, x, y)=c_{2}^{-1} & \mathrm{e}^{\frac{-\delta(x-u)}{2 c_{2}}}\left[\mathbf{I}-{ }^{x-u} \mathbf{\Psi}_{c_{2}}(\delta / 2) \cdot{ }^{u} \mathbf{\Psi}_{u-b, c_{2}, c_{1}}^{r}(\delta / 2)\right]^{-1} \\
\cdot & { }_{0} \hat{\mathbf{f}}_{11, c_{2}}(0, x-u, \delta / 2)\left[\mathbf{I}-\mathbf{\Psi}_{c_{2}}(\delta / 2) \cdot{ }^{x} \mathbf{\Psi}_{x-b, c_{2}, c_{1}}^{r}(\delta / 2)\right]^{-1} \\
\cdot & \mathbf{T}_{12} \mathrm{e}^{\mathbf{T}_{22,1}(x+y)} \mathbf{t}_{21,1} .
\end{aligned}
$$


(b) for $b<x<u$ and $y>0$,

$$
\begin{aligned}
\mathbf{g}_{u}^{b}(\delta, x, y)=c_{2}^{-1} \rho_{\delta, c_{2}}(u-x) \cdot{ }^{x} \mathbf{\Psi}_{x-b, c_{2}, c_{1}}^{r}(\delta / 2) \\
\cdot\left[\mathbf{I}-\Psi_{c_{2}}(\delta / 2) \cdot{ }^{x} \boldsymbol{\Psi}_{x-b, c_{2}, c_{1}}^{r}(\delta / 2)\right]^{-1} \cdot \mathbf{T}_{12} \mathrm{e}^{\mathbf{T}_{22,1}(x+y)} \mathbf{t}_{21,1} .
\end{aligned}
$$

(c) for $0<x<b$ and $y>0$,

$$
\begin{gathered}
\mathbf{g}_{u}^{b}(\delta, x, y)=c_{1}^{-1} \rho_{\delta}^{b-x}(u-x) \cdot{ }^{x} \boldsymbol{\Psi}_{c_{1}}^{r}(\delta / 2)\left[\mathbf{I}-\boldsymbol{\Psi}_{b-x, c_{1}, c_{2}}(\delta / 2) \cdot{ }^{x} \boldsymbol{\Psi}_{c_{1}}^{r}(\delta / 2)\right]^{-1} \\
\cdot \mathbf{T}_{12} \mathrm{e}^{\mathbf{T}_{22,1}(x+y)} \mathbf{t}_{21,1} .
\end{gathered}
$$

The proof of Theorem 3 follows along the same line of logic as Theorem 2. We remark that the Laplace transforms of the time to ruin $\rho_{\delta, c_{2}}(u)$ and $\rho_{\delta}^{b-x}(u)$ that appear in Theorem 3 are given in Section 3 of Badescu et al. [23, (2007)].

This completes the analysis of the discounted density $\mathbf{g}_{u}^{b}(\delta, x, y)$ in the single threshold surplus process $\left\{R^{b}(t), t \geq 0\right\}$. Extensions to the more general multi-threshold dividend strategy have been considered by many authors (e.g. Albrecher and Hartinger [7, (2007)], Lin and Sendova [47, (2008)], Yang and Zhang [61, (2008)] and Zhou [62, (2006)]). We refer the interested reader to Badescu et al. [23, (2007)] for the identification of the discounted density of the surplus prior to ruin and the deficit at ruin in a multi-threshold MAP risk model.

\section{Other applications and concluding remarks}

So far, our discussion of MAMs applications in ruin theory have gravitated around the analysis of the discounted joint density of the surplus immediately prior to ruin and the deficit at ruin. This discounted joint density is of interest by itself and also plays a vital role in Gerber-Shiu type analysis of a given surplus process. However, there exist many more applications of MAMs in ruin theory. Thus, for the reminder of this review paper, we aim at taking a broader view at the literature by listing other applications of MAMs in a ruin theoretical context.

A relatively new application of MAMs in ruin theory is the so-called Erlangization method that has been mainly developed for the evaluation of finite-time ruin probabilities. The idea was first introduced by Avram and Usabel [18, (2003)] where the concept of ruin before an exponentially-distributed random horizon is considered in the compound Poisson risk model with phase-type claim sizes. This idea was further extended by Asmussen et al. [12, (2002)] to the case of ruin before a phase-type random horizon. For an Erlang-distributed random horizon with a mean equal to the desired finite-time span, an approximation to the finite-time ruin probability is obtained. Furthermore, Asmussen et al. [12, (2002)] proved that this approximation converges to the exact finite-time ruin probability as the shape parameter of the Erlang distribution approaches infinity. The Erlangization idea is further used to analyze the general Sparre Andersen risk model (see e.g. Stanford et al. [59, (2005)]) and the Markovian fluid flow model (see e.g. Ramaswami et al. [56, (2008)]).

Risk models with perturbations have also been studied using MAMs. In the literature, the analysis of these perturbed risk models have preferred the spectral decomposition type-analysis to the sample path analysis presented in Section 3. Asmussen [10, (1995)] studied the stationary distribution of a fluid flow model with paths which are piecewise linear or Brownian, with constant drifts and variances given by an underlying Markov process. Based on a vector-valued martingale introduced by Asmussen and Kella [13, (2000)], Badescu and Breuer [19, (2008)] analyzed the LST of the time to ruin in a MAP risk model with perturbation and phase-type downward jumps. Further generalizations of MAP risk processes with both downward and upward phase-type distributed jumps can be found in Breuer [29, (2008)]. Among other things, a numerically stable iteration is proposed to compute the LST of certain first passage times. Also a generalization of the discounted joint density of the surplus prior to ruin and the deficit at ruin is considered in Breuer [30, (2009)]. In particular a quintuple law that also includes the minimum surplus level before 
ruin, the time to reach this minimum and the remaining time until ruin is derived under a Markov additive process with phase-type jumps.

Risk models modified by various dividend payment strategies have also been another active research topic in ruin theory. The reader is referred to Avanzi [17, (2009)] for a comprehensive and thorough review on this topic. Capitalizing on the same MAMs developed for the study of fluid queues, Badescu et al. [23, 24, (2007)] have obtained expressions for the expected discounted dividend payments before ruin in a MAP risk model in the presence of a threshold dividend strategy and a multi-threshold dividend strategy respectively. For the higher-order moments of the discounted dividend payments, a recursive scheme was later proposed by Badescu and Landriault [25, 26, (2007, 2008)] to allow their calculation. In these papers a recursive algorithm is developed to compute the moments of the discounted dividend payments. The starting point of the recursive procedure is the calculation of the moments of the discounted dividend payments in a threshold-free surplus process consisting of only the top layer of the risk process. The recursion is constructed from top to bottom by adding the next lower layer at each iteration. The reader is also referred to a discussion by Cheung [31, (2008)] where the dual MAP risk model is studied and the associated recursive scheme is elegantly derived.

A recent extension of the Sparre Andersen insurance risk model for which the joint distribution of the interclaim time and the subsequent claim size is bivariate phase-type (see Assaf et al. [16, (1984)]) is considered in Badescu et al. [22, (2009)]. Relying on the existing connection between risk processes and fluid flows, the authors construct an analytically tractable fluid flow that leads to the analysis of various ruin-related quantities including the Gerber-Shiu discounted penalty function when the penalty function depends on the deficit at ruin only.

To conclude, we believe that the connection between surplus processes and fluid flows has been instrumental to the literature in ruin theory. Capitalizing on the development of MAMs for fluid flows, this connection has opened up new research directions to analyze different ruin-related problems. More importantly, it provides an alternative to the traditional analytic approach commonly used in ruin theory. Having both their own strengths and weaknesses, it is our belief that the combination of these two approaches will result in a better understanding of the risks inherent to the event "ruin" for an insurer.

\section{References}

[1] Ahn, S. And BAdescu, A. L., (2007). On the analysis of the Gerber-Shiu discounted penalty function for risk processes with Markovian arrivals, Insurance Math. Econom., 41(2), 234-249.

[2] Ahn, S., Badescu, A. L. And Ramaswami, V., (2007). Time dependent analysis of finite buffer fluid flows and risk models with a dividend barrier, Queueing Syst., 55(4), 207-222.

[3] Ahn, S. And Ramaswami, V., (2004). Transient analysis of fluid flow models via stochastic coupling to a queue, Stoch. Models, 20(1), 71-101.

[4] Ahn, S. AND RAmASWAMi, V., (2005). Efficient algorithms for transient analysis of stochastic fluid flow models, J. Appl. Probab., 42(2): 531-549.

[5] Ahn, S. And Ramaswami, V., (2006). Transient analysis of fluid flow models via elementary level crossing arguments, Stoch. Models, 22, 1, 129-147.

[6] Albrecher H. And Boxma O., (2005). On the discounted penalty function in a Markov dependent risk model, Insurance Math. Econom., 37(3), 650-672.

[7] Albrecher, H. And Hartinger, J., (2007). A risk model with multi-layer dividend strategy, N. Am. Actuar. J., 11(2), 43-64.

[8] Albrecher, H., Hartinger, J. And Thonhauser, S., (2006). On exact solutions for dividend strategies of threshold and linear barrier type in a Sparre Andersen model, Astin Bull., 37(2), 203-233. 
[9] Albrecher, H. And Teugels, J. L., (2006). Exponential behavior in the presence of dependence in risk theory. J. Appl. Probab., 43(1), 257-273.

[10] Asmussen, S., (1995). Stationary distributions via first passage times, Advances in Queueing: Theory, Methods, and Open Problems, 79-102.

[11] Asmussen, S., (2000). Ruin Probabilities, World Scientific, Singapore.

[12] Asmussen, S., Avram, F. And Usabel, M., (2002). Erlangian approximations for finite-horizon ruin probabilities, Astin Bull., 32(2), 267-281.

[13] Asmussen, S. And Kella, O., (2000). A multi-dimensional martingale for Markov additive processes and its applications, Adv. in Appl. Probab., 32, 376-393.

[14] Asmussen, S. And Koole, G., (1993). Marked point processes as limits of Markovian arrival streams, J. Appl. Probab., 30, 365-372.

[15] Asmussen, S., Nerman, O. And Olsson,M., (1996). Fitting phase-type distributions via the EM algorithm, Scand. J. Statist., 23(4), 419-441.

[16] Assaf, David, Langberg, Naftali, A., Savits, Thomas H. and Shaked, Moshe, (1984). Multivariate phase-type distributions, Oper. Res., 32(3), 688-702.

[17] AvAnZI, B., (2009). Strategies for dividend distribution: a review, N. Am. Actuar. J.. In press.

[18] Avram, F. And Usabel, M., (2003). Finite time ruin probabilities with one Laplace inversion, Insurance Math. Econom., 32, 371-377.

[19] BAdescu, A. L. AND BReuer, L., (2008). The use of vector-valued martingales in risk theory, Blatter der DGVFM, 29, 1-12.

[20] Badescu, A. L., Breuer, L., Da Silva Soares, A., Latouche, G., Remiche, M.-A. And StanFORD, D., (2005). Risk processes analyzed as fluid queues, Scand. Actuar. J., 2005(2), 127-141.

[21] Badescu, A. L., Breuer, L., Drekic, S., Latouche, G. And Stanford, D. A., (2005). The joint density of the surplus prior to ruin and the deficit at ruin for a correlated risk process, Scand. Actuar. J., 2005(6), 433-446.

[22] Badescu, A. L., Cheung, E. C. K. And Landriault, D., (2009). Dependent risk models with bivariate phase-type distributions, J. Appl. Probab., 46(1), 113-131.

[23] Badescu, A. L., Drekic, S. And Landriault, D., (2007). Analysis of a threshold dividend strategy for a MAP risk model, Scand. Actuar. J., 2007(4), 227-247.

[24] Badescu, A. L., Drekic, S. And Landriault, D., (2007). On the analysis of a multi-threshold Markovian risk model, Scand. Actuar. J., 2007(4), 248-260.

[25] Badescu, A. L. And Landriault, D., (2007). Moments of the discounted dividends in a threshold-type Markovian risk process, Braz. J. Probab. Stat., 21, 13-25.

[26] Badescu, A. L. And Landriault, D., (2008). Recursive calculation of the dividend moments in a multithreshold risk model, N. Am. Actuar. J., 12(1), 74-88.

[27] Bean, N. G., Malgorzata, M. O. and Taylor, P. G., (2005). Hitting probabilities and hitting times for stochastic fluid flows, Stochastic Process. Appl., 11, 1530-1556.

[28] Boudreault, M., Cossette, H., Landriault, D. and Marceau, E., (2006). On a risk model with dependence between interclaim arrivals and claim sizes, Scand. Actuar. J., 2006(5), 265-285.

[29] BREUER, L., (2008). First passage times for Markov-additive processes with positive jumps of phase-type. J. Appl. Probab., 45(3), 779-799.

[30] Breuer, L., (2009). A quintuple law for Markov-additive processes with phase-type jumps, submitted. 
[31] Cheung, E. C. K., (2008). Discussion of "Recursive calculation of the dividend moments in a multi-threshold risk model". N. Am. Actuar. J., 12(3), 336-340.

[32] Cheung, E. C. K. And Landriault, D., (2009). Perturbed MAP risk models with dividend barrier strategies, J. Appl. Probab., in press.

[33] Cheung, E. C. K., Landriault, D., Willmot, G. E. And Woo, J.-K., (2009). Gerber-Shiu analysis with a generalized penalty function, Scand. Actuar. J., in press.

[34] Cossette, H., Marceau, E. And Marri, F., (2008). On the compound Poisson risk model with dependence based on a generalized Farlie-Gumbel-Morgenstern copula, Insurance Math. Econom., 43(3), 444-455.

[35] Dickson, D. C. M., (1992). On the distribution of the surplus prior to ruin, Insurance Math. Econom., 11(3), 191-207.

[36] Dickson, D. C. M., (1993). On the distribution of the claim causing ruin, Insurance Math. Econom., 12, 143154.

[37] Dickson, D. C. M. And Hipp, C., (1998). Ruin probabilities for Erlang(2) risk process, Insurance Math. Econom., 22, 251-262.

[38] Dufresne, F. And Gerber, H. U., (1988). The surpluses immediately before and at ruin, and the amount of the claim causing ruin, Insurance Math. Econom., 7, 193-199.

[39] Gerber, H. U. And ShiU, E. S. W., (1997). The joint distribution of the time of ruin, the surplus immediately before ruin and the deficit at ruin, Insurance Math. Econom., 21(2), 129-137.

[40] Gerber H. U. And Shiu E. S. W., (1998). On the time value of ruin, N. Am. Actuar. J., 2(1) 48-78.

[41] Gerber H. U., Shiu E. S. W., (2005). The time value of ruin in a Sparre-Andersen model, N. Am. Actuar. J., 9, 49-84.

[42] Landriault, D. And Willmot, G. E., (2009). On the joint distributions of the time to ruin, the surplus prior to ruin and the deficit at ruin in the classical risk model, N. Am. Actuar. J., in press.

[43] Latouche, G. And Ramaswami, V., (1993). A logarithmic reduction algorithm for quasi-birth-and-death processes, J. Appl. Probab., 30, 650-674.

[44] Latouche, G. And Ramaswami, V., (1999). Introduction to Matrix Analytic Methods in Stochastic Modeling, ASA SIAM, Philadelphia.

[45] Li, S. ANd GARrido, J., (2005). On a general class of renewal risk process: analysis of the Gerber-Shiu function, Advances in Applied Probabilities, 37 (3), 836-856.

[46] Lin, X. S. And Pavlova, K., (2005). The compound Poisson risk model with a threshold dividend strategy, Insurance Math. Econom., 38(1), 57-80.

[47] Lin, X. Sheldon And Sendova, Kristina, (2008). The compound Poisson risk model with multiple thresholds, Insurance Math. Econom., 42, 617-627.

[48] Lin X. S. And Willmot, G. E., (1999). Analysis of a defective renewal equation arising in ruin theory, Insurance Math. Econom., 25(1), 63-84.

[49] LU, Y. AND LI, S., (2009). The Markovian regime-switching risk model with a threshold dividend strategy, Insurance Math. Econom., 44(2), 296-303.

[50] Lu, Y. And Tsai, C. C. L., (2007). The expected discounted penalty at ruin for a Markov-modulated risk process perturbed by diffusion, N. Am. Actuar. J., 11(2), 136-152.

[51] Neuts, M., (1978). Renewal processes of phase-type, Naval Res. Logist., 25, 445-454.

[52] Neuts, M., (1979). A versatile markovian point process, J. Appl. Probab., 16(4), 764-779. 
[53] Neuts, M., (1981). Matrix-geometric solutions in stochastic models, DOVER Publications, New York.

[54] Ramaswami, V., (1999). Matrix analytic methods for stochastic fluid flows. Teletraffic Engineering in a Competitive World (Proceedings of the 16th International Teletraffic Congress), 1019-1030.

[55] Ramaswami, V., (2006). Passage times in fluid models with application to risk processes, Methodol. Comput. Appl. Probab., 8(4), 497-515.

[56] Ramaswami, V., Woolford, D. G. And Stanford, D. A., (2008). The erlangization method for Markovian fluid flows, Ann. of Oper. Res., 160(1), 215-225.

[57] Ren, J., (2007). The discounted joint probability of the surplus prior to ruin and the deficit at ruin, $N$. Am. Actuar. J., 11(3), 128-136.

[58] Rogers, L. C. G., (1994). Fluid models in queueing theory and Wiener-Hopf factorization of Markov chains, Ann. Appl. Probab., 4, 390-413.

[59] Stanford, D. A., Avram, F., Badescu, A. L., Breuer, L., Soares, A. S. and Latouche, G., (2005). Phase-type approximations to finite-time ruin probabilities in the Sparre-Andersen and stationary renewal risk models, Astin Bull., 35(1), 131-144.

[60] Wu, R., WANG, G. AND WEI, L, (2003). Joint distributions of some actuarial random vectors containing the time of ruin, Insurance Math. Econom., 33(1), 147-161.

[61] YAng, H. AND Zhang, Z, (2008). Gerber-Shiu Discounted penalty function in a Sparre Andresen model with mutli-layer dividend strategy, Insurance Math. Econom., 42(3), 984-991.

[62] ZHOU, XIAOWEN, (2006). Classical risk model with a multi-layer premium rate, preprint.

\section{A Appendix}

Consider a QBD consisting of three repeating square blocks $\mathbf{A}_{0}, \mathbf{A}_{1}, \mathbf{A}_{2}$ which represents the transition matrices one level up, in the same level, and a level below respectively. Assume now that the process is observed when the level changes. The probability that the level decreases by 1 after $n$ iterations is given by $\mathbf{A}_{1}^{n} \mathbf{A}_{2}$. Taking the sum after all possible iterations, one obtains the probability of eventually going one level down as $\mathbf{B}_{2}=\left(\mathbf{I}-\mathbf{A}_{1}\right)^{-1} \mathbf{A}_{2}$. In the similar fashion the matrix of probabilities to eventually go a level up is $\mathbf{B}_{0}=\left(\mathbf{I}-\mathbf{A}_{1}\right)^{-1} \mathbf{A}_{0}$. In this way it is possible to obtain a new Markov chain with a transition matrix with $\mathbf{0}$ block matrices on the main-diagonal, $\mathbf{B}_{0}$ on the upper main diagonal and $\mathbf{B}_{2}$ on the lower main diagonal. In this new created Markov chain it is easy to see that:

$$
\mathbf{Y}=\mathbf{B}_{2}+\mathbf{B}_{0} \mathbf{Y}^{2}
$$

(To verify, substitution for $\mathbf{B}_{2}$ and $\mathbf{B}_{0}$ and rearrangement yields (4). Suppose now that the process starts in an even level, for example when the insurer receives premiums. At the next level, the process will be automatically in an odd level (e.g. a claim payment period), indifferent if the step is up or down. This observation makes it possible to look at the process precisely 2 steps from now. If one observes the process only at even times, the process will be necessarily in an even level. If the process goes up, it must go up two steps, so instead of $\mathbf{Y}$, one will have $\mathbf{Y}^{(2)}=\mathbf{Y}^{2}$. This new formed process is again a QBD. The matrix that gives the probability to go two levels down is $\mathbf{B}_{2}^{2}$ and two levels up is $\mathbf{B}_{0}^{2}$. The new matrix of transitions between levels has now the form:

$$
\left[\begin{array}{ccccc}
* & * & \mathbf{0} & \mathbf{0} & \ldots \\
& \mathbf{A}_{1}^{(1)} & \mathbf{A}_{0}^{(1)} & \mathbf{0} & \ldots \\
\mathbf{0} & \mathbf{A}_{2}^{(1)} & \mathbf{A}_{1}^{(1)} & \mathbf{A}_{0}^{(1)} & \ldots \\
\mathbf{0} & \mathbf{0} & \mathbf{A}_{2}^{(1)} & \mathbf{A}_{1}^{(1)} & \ldots \\
\vdots & \vdots & \vdots & \vdots & \ddots
\end{array}\right]
$$


with $\mathbf{A}_{0}^{(1)}=\mathbf{B}_{0}{ }^{2}, \mathbf{A}_{2}^{(1)}=\mathbf{B}_{2}{ }^{2}$ and $\mathbf{A}_{1}^{(1)}=\mathbf{B}_{0} \mathbf{B}_{2}+\mathbf{B}_{2} \mathbf{B}_{0}$. As mentioned before, the matrix $\mathbf{Y}$ that corresponds to the new process is the matrix $\mathbf{Y}^{(2)}$ of the old process, so equation (20) applies with $\mathbf{Y}$ replaced by $\mathbf{Y}^{(2)}$, and $\mathbf{B}_{0}$ and $\mathbf{B}_{2}$ replaced by $\mathbf{B}_{0}^{(1)}$ and $\mathbf{B}_{0}^{(1)}$ given by:

$$
\begin{aligned}
& \mathbf{B}_{0}{ }^{(1)}=\left(\mathbf{I}-\mathbf{A}_{1}{ }^{(1)}\right)^{-1} \mathbf{A}_{0}{ }^{(1)} \\
& \mathbf{B}_{2}{ }^{(1)}=\left(\mathbf{I}-\mathbf{A}_{1}{ }^{(1)}\right)^{-1} \mathbf{A}_{2}{ }^{(1)}
\end{aligned}
$$

Equation (20) becomes:

$$
\mathbf{Y}^{(2)}=\mathbf{B}_{2}^{(1)}+\mathbf{B}_{0}^{(1)}\left(\mathbf{Y}^{(2)}\right)^{2}
$$

Since $\mathbf{Y}^{(2)}=\mathbf{Y}^{2}$, one can further obtain:

$$
\mathbf{Y}=\mathbf{B}_{2}+\mathbf{B}_{0} \mathbf{B}_{2}^{(1)}+\mathbf{B}_{0} \mathbf{B}_{0}^{(1)}\left(\mathbf{Y}^{(2)}\right)^{2}
$$

If one continues in the same fashion, one can obtain a new Markov chain with $\mathbf{Y}^{(4)}=\mathbf{Y}^{(2)}{ }^{2}=\mathbf{Y}^{4}$ and so on. Continuing the iterations, the matrix $\mathbf{Y}$ will converge quadratically fast to

$$
\mathbf{Y}=\sum_{k=0}^{\infty}\left(\prod_{i=0}^{k-1} \mathbf{B}_{0}{ }^{(i)}\right) \mathbf{B}_{2}{ }^{(k)},
$$

where

$$
\mathbf{B}_{i}^{(0)}=\left(\mathbf{I}-\mathbf{A}_{1}\right)^{-1} \mathbf{A}_{i}, \quad i=0,2 .
$$

and

$$
\mathbf{B}_{i}{ }^{(k+1)}=\left(\mathbf{I}-\mathbf{A}_{1}{ }^{(k+1)}\right)^{-1} \mathbf{A}_{i}^{(k+1)}=\left(\mathbf{I}-\mathbf{B}_{0}{ }^{(k)} \mathbf{B}_{2}{ }^{(k)}-\mathbf{B}_{2}{ }^{(k)} \mathbf{B}_{0}{ }^{(k)}\right)^{-1}\left(\mathbf{B}_{i}{ }^{(k)}\right)^{2}, \quad i=0,2 .
$$

Once the matrix $\mathbf{Y}$ is obtained, the upper-right block matrix is $\mathbf{\Psi}(\delta)$.

Andrei L. Badescu
Department of Statistics,
University of Toronto,
100 St. George St.,
Toronto, Ontario, Canada

\section{David Landriault}

Department of Statistics and Actuarial Science, University of Waterloo, 200

University Avenue West,

Waterloo, Ontario, Canada 